\title{
An Analytical Error Model for Spaceborne SAR Multichannel Azimuth Reconstruction
}

\author{
Felipe Queiroz de Almeida, Marwan Younis, Senior Member, IEEE, \\ Gerhard Krieger, Fellow, IEEE and Alberto Moreira, Fellow, IEEE
}

\begin{abstract}
In the context of spaceborne synthetic aperture radar (SAR) for remote sensing, multichannel system architectures coupled with digital beamforming (DBF) techniques are deemed a necessary technological advancement to fulfil the requirements for near future spaceborne radar missions. Calibration of such systems is an important topic, since channel imbalances may lead to considerable degradation of their performance. This paper analyzes the impact of residual errors in a SAR system with multiple channels in azimuth and derives an analytical model for the resulting performance degradation, which may be used in system design as an aid to establish requirements in an error budget analysis.
\end{abstract}

Index Terms - Channel imbalances, Digital beamforming, Error modeling, High resolution wide swath, Multichannel SAR, Spaceborne radar, Synthetic aperture radar

\section{INTRODUCTION}

$\mathrm{S}_{\mathrm{p}}^{\mathrm{p}}$ PACEBORNE synthetic aperture radar (SAR) [1] data currently enjoy an increasing acceptance in the scientific community, owing to its myriad applications. Imaging a wide swath with a high spatial resolution (HRWS) [2], [3] - which is necessary to provide a broad and up-to-date coverage of high quality data - is however a fundamental problem in SAR system design [1], since single channel systems are subject to a well-known compromise between azimuth resolution and coverage [4].

The usage of multichannel architectures (especially in azimuth) and digital beamforming (DBF) techniques [5], [6] poses a promising solution to this dilemma and is currently subject of technological development for implementation of Sentinel-1 Next Generation and other HRWS satellite missions [7], [8]. A basic block diagram of the concept, which is based on multichannel sampling in azimuth [9], is provided in Fig. 1.

The signal processing for this class of system relies, however, on the knowledge of the receive channels' transfer functions [6], which makes adequate channel calibration crucial, as channel imbalances may severely degrade performance [10]. In terms of system design, this poses the

Manuscript received 17.08.2017. F. Queiroz de Almeida, M. Younis, G. Krieger and A. Moreira are with the Microwaves and Radar Institute of the German Aerospace Center (DLR), Oberpfaffenhofen, Germany (e-mail: felipe.queirozdealmeida@dlr.de.).

Part of this work was developed in the frame of the European Space Agency (ESA) project "Data Reduction for Digital Beamforming Instruments" ESA ITT AO/1-8469/15/NL/FE. problem of how to specify radar electronics and calibration accuracy requirements, which in turn requires an understanding of the impact of such errors over performance. This paper analyses the impact of residual channel imbalances on the processing of a system with multiple azimuth channels and derives an analytical model for the degradation of the performance in comparison to what is expected in the errorfree scenario. Sections II and III provide the signal model and mathematical derivation, whereas Section IV presents simulation results to validate the established model. Finally, Section V provides a summary and discussion of the material.

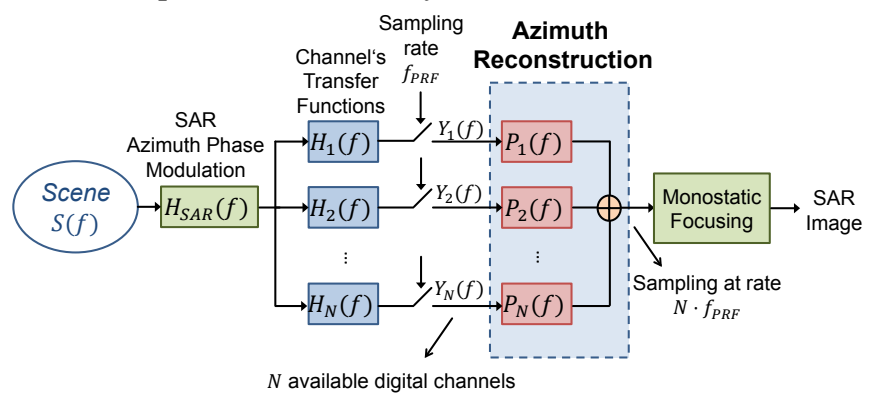

Fig. 1: Block diagram describing the data acquisition and processing in a SAR system with multiple azimuth channels. The scene's backscattered signal is acquired by $N$ receivers, modelled by their transfer functions $H_{k}(f)$, at a (typically sub-Nyquist) rate of $f_{\mathrm{PRF}}$. The signal processing (digital filters $\left.P_{k}(f)\right)$ restores sampling to $N \cdot f_{\mathrm{PRF}}$, such that focusing yields a SAR image equivalent to a single-channel system sampled directly at $N \cdot f_{\mathrm{PRF}}$.

\section{MUltichanNEL AZIMUTH RECONSTRUCTION AND THE EFFECT OF CHANNEL IMBALANCES}

\section{A. Signal Model: Error-free Case}

In the following, a system with one $\mathrm{Tx}$ and $N \mathrm{Rx}$ azimuth channels is considered. As described in [6] in detail, if the sampling of each of the individual channels occurs at a rate of $f_{P R F}$, the effective sampling of the equivalent monostatic system is $N \cdot f_{P R F}$, as $N$ samples are recorded for each received pulse. Thus, a complex signal spectrum $U(f)$ of Doppler bandwidth $B_{D} \leq N \cdot f_{\mathrm{PRF}}$ may be recovered unambiguously by proper combination of the aliased spectra of each of the channels in the frequency domain.

Taking the limiting case, the total signal bandwidth of $N \cdot f_{P R F}$ is divided into $N$ contiguous sub-bands

$I_{m}=\left[-N \cdot \frac{f_{\mathrm{PRF}}}{2}+(m-1) \cdot f_{\mathrm{PRF}},-N \cdot \frac{f_{\mathrm{PRF}}}{2}+m \cdot f_{\mathrm{PRF}}[\right.$,

each of length $f_{\mathrm{PRF}}$, for $1 \leq m \leq N$. Due to the aliasing, the signal spectrum of the channels may be represented in any interval 
of length $f_{\mathrm{PRF}}$, taken here to be $I_{1}$. The $k$-th azimuth channel is considered to be described by the transfer function $H_{k}\left(f+(m-1) \cdot f_{\mathrm{PRF}}\right)$, with $1 \leq m, k \leq N$. The complete spectrum $U(f)$ of the scene to be recovered may be divided into $N$ signals $S_{m}(f)=U\left(f+(m-1) \cdot f_{\mathrm{PRF}}\right)$, for $f$ in $I_{1}$. These signals can be regarded as azimuth looks of the SAR image, in accordance with typical SAR processing nomenclature.

Considering that the $k$-th azimuth channel is positioned at $\Delta x_{k}$ and that the platform velocity in along-track is $V_{P}$, the channel transfer function in frequency domain after a Taylor expansion may be approximated by [6]

$H_{k}(f)=e^{-j \cdot \Delta \phi_{k}} \cdot e^{-j \cdot 2 \cdot \pi \cdot \Delta t_{k} \cdot f}$,

where $\Delta t_{k}=\frac{\Delta x_{k}}{2 \cdot V_{P}}$ are the delays induced by the phase center baselines and $\Delta \phi_{k}$ is a constant phase.

Taking into account the sub-band division, the multichannel system in frequency domain may be described by the $N$ x $N$ matrix $\boldsymbol{H}(f)$ with elements $H_{k l}(f)=H_{k}\left(f+(l-1) \cdot f_{\mathrm{PRF}}\right)$ and the sub-sampled signal at each channel $k$ by

$y_{k}(f)=\sum_{m=1}^{N} H_{k}\left(f+(m-1) \cdot f_{\mathrm{PRF}}\right) \cdot S_{m}(f)$,

so that, in matrix notation,

$\boldsymbol{y}(f)=\boldsymbol{H}(f) \cdot \boldsymbol{s}(f)$,

where $\boldsymbol{y}(f)=\left[y_{1}(f), y_{2}(f), \ldots, y_{N}(f)\right]^{T}$ and

$\boldsymbol{s}(f)=\left[S_{1}(f), S_{2}(f), \ldots, S_{N}(f)\right]^{T}$.

Reconstruction can be regarded as an estimator

$\hat{\boldsymbol{s}}(f)=\boldsymbol{P}(f) \cdot \boldsymbol{y}(f)$,

where the filter matrix has elements

$P_{m k}(f)=P_{k}\left(f+(m-1) \cdot f_{\mathrm{PRF}}\right)$

and hence each look $S_{m}(f)$ is recovered by means of row $m$ of matrix $\boldsymbol{P}(f)$, and each column of it is applied to a particular channel $k$. In particular, $\boldsymbol{P}(f)=\boldsymbol{H}(f)^{-1}$ yields ideal reconstruction of the bandlimited signal in the noiseless case and can be shown to be optimal in a mean square error (MSE) sense, even in the presence of noise and a non-bandlimited spectrum [11], [12]. Alternative reconstruction schemes are analyzed in [12], [13], and amount to choosing the reconstruction filters $\boldsymbol{P}(f)$ following different strategies, e.g. setting as design goal the suppression of white noise alongside the reconstruction of the signal.

\section{B. Error Model}

The signal model in Section II.A may be augmented to encompass channel errors by considering that the system is actually described by a channel matrix $\boldsymbol{H}_{\text {error }}(f)$ which differs from the nominal channel matrix $\boldsymbol{H}(f)$. Thus a modelling error is assumed, which may be caused by residual channel imbalances introduced by a previous beamforming stage (e.g. synchronization errors between the azimuth channels, clock drifts for the ADCs and amplitude and phase drifts in the Transmit/Receive (T/R) Modules).

Let each of the $N$ channels be affected by a phase error $\xi_{i}$, and a normalized amplitude error $\epsilon_{i}, 1 \leq i \leq N$. We assume $\epsilon_{i}$ to be independent identically distributed (i.i.d.) random variables following a Gaussian distribution $N\left(0, \sigma_{\epsilon}^{2}\right)$, whereas two models are considered for the phase errors. First, a uniform distribution in the interval $\left[-\frac{\xi_{u}}{2}, \frac{\xi_{u}}{2}\right]$ is considered, as a pessimistic assumption on the spread of the phases, while still allowing for a maximum error level. This could for example be due to the phase shift quantization in temperature-compensated T/R-Modules (e.g. 6 bit phase shifters such as used in TerraSAR-X lead to a precision of $\pm 5.6^{\circ}$ ). Such a model is convenient for establishing system design and calibration requirements, as only the maximum error needs to be specified from the analysis of the previous processing stages. Second, small phase errors (without a predefined distribution) are considered, i.e., satisfying the condition

$\left(1+\epsilon_{k}\right) \cdot \exp \left(j \cdot \xi_{k}\right)-1 \cong \epsilon_{k}+j \cdot \xi_{k}$,

a model which is interesting for its simplicity and generality.

In both cases, the amplitude and phase errors are assumed to be independent from each other and from the signal ${ }^{1}$. Then, the actual system matrix becomes

$\boldsymbol{H}_{\text {error }}(f)=\boldsymbol{D} \cdot \boldsymbol{H}(f)$

where the diagonal, frequency independent ${ }^{2}$ error matrix has elements

$\boldsymbol{D}=\operatorname{diag}\left(1+\epsilon_{i}\right) \cdot \operatorname{diag}\left(\exp \left(j \cdot \xi_{i}\right)\right)$.

Reconstruction (cf. (4)) of the signal from the system described by (6) with the nominal reconstruction filters ${ }^{3} \boldsymbol{P}(f)$ yields

$\hat{\boldsymbol{s}}(f)=\boldsymbol{P}(f) \cdot(\boldsymbol{D} \cdot \boldsymbol{H}(f)) \cdot \boldsymbol{s}(f)$,

or, by expressing $\boldsymbol{D}=\boldsymbol{I}+(\boldsymbol{D}-\boldsymbol{I})$,

$\hat{\boldsymbol{s}}(f)=\boldsymbol{s}_{\text {error-free }}(f)+\boldsymbol{P}(f) \cdot(\boldsymbol{D}-\boldsymbol{I}) \cdot \boldsymbol{H}(f) \cdot \boldsymbol{s}(f)$

$\hat{\boldsymbol{s}}(f)=\boldsymbol{s}_{\text {error-free }}(f)+\boldsymbol{s}_{\text {error }}(f)$,

where $\boldsymbol{s}_{\text {error-free }}(f)$ is the result of reconstruction in the absence of errors. Note that in general $\boldsymbol{s}_{\text {error-free }}(f) \neq \boldsymbol{s}(f)$, unless the signal is band-limited and noise free, and ambiguities cannot be perfectly suppressed, i.e.

$\boldsymbol{s}_{\text {error-free }}(f)=\boldsymbol{s}(f)+\boldsymbol{s}_{\text {amb }}(f)$,

where $\boldsymbol{s}_{\mathrm{amb}}(f)$ denotes the residual ambiguities.

Due to the assumption that the zero-mean errors are independent of the signal (and also of additive noise), $E\left[\boldsymbol{s}_{\text {error-free }}(f) \cdot \boldsymbol{s}_{\text {error }}(f)^{H}\right]=0$ and thus the ambiguous power due to them simply adds to that due to other factors, i.e.

$E\left[\|\hat{\boldsymbol{s}}(f)\|^{2}\right]=E\left[\left\|\boldsymbol{s}_{\text {error-free }}(f)\right\|^{2}\right]+E\left[\left\|\boldsymbol{s}_{\text {error }}(f)\right\|^{2}\right]$.

The average signal power remains the same, so the final Azimuth Ambiguity-to-Signal Ratio (AASR) in the presence of errors following the model described in this section is

$A A S R=A A S R_{\text {error-free }}+A A S R_{\text {errors }}$,

i.e. an additive term degrading the error-free result obtained for a particular (and not necessarily ideal) configuration.

As will be elaborated, the statistical approach adopted in this paper is to assume a large set of calibration errors for the channels and then estimate the ensemble average of (14) as an indication of the AASR to be expected in a typical operational scenario.

\footnotetext{
${ }^{1}$ A model also usual in describing quantization errors, meaning the results can be extended to this case.

${ }^{2}$ We choose to present the simplest model for increased clarity, though frequency dependency of the errors within an interval of width $f_{\mathrm{PRF}}$ can be seamlessly incorporated into the derivation up to (26).

${ }^{3}$ Notice that $\boldsymbol{P}(f)=\boldsymbol{H}(f)^{-1}$ as in [6] is a possible choice, but the derivation holds for arbitrary reconstruction filters $\boldsymbol{P}(f)$.
} 


\section{DeRIVATION Of Residual AMBIGUITY LEVEL DUE TO ERRORS}

Taking the reconstruction error $\boldsymbol{\Delta} \boldsymbol{s}(f)=\hat{\boldsymbol{s}}(f)-\boldsymbol{s}(f)$ from (11), the quantity of interest is the residual ambiguous power density caused by the errors, that is $\sigma_{\text {error }}^{2}(f)=E\left[\|\boldsymbol{\Delta} \boldsymbol{s}(f)\|^{2}\right]$. To calculate it, we introduce the auxiliary random variables

$\beta_{k}=\left(1+\epsilon_{k}\right) \cdot \exp \left(j \cdot \xi_{k}\right)-1 ; \quad$ for $1 \leq k \leq N$,

which are pairwise independent and, from (11) and (4), allow the components of the error vector to be written as

$\Delta s_{m}(f)=\sum_{k=0}^{N} P_{m k}(f) \cdot \beta_{k} \cdot y_{k}(f)$.

One may then write, using (16) and rearranging,

$\sigma_{\text {error }}^{2}(f)=\sum_{m=0}^{N} E\left[\left|\Delta s_{m}(f)\right|^{2}\right]$

$\sigma_{\text {error }}^{2}(f)=\sum_{m=0}^{N} \sum_{k=0}^{N} E\left[\left|y_{k}(f)\right|^{2}\right] \cdot E\left[\left|\beta_{k}\right|^{2}\right] \cdot\left|P_{m k}(f)\right|^{2}$.

Since the signal components in the $N$ x 1 vector $\boldsymbol{s}(f)$ represent non-overlapping azimuth looks of the Doppler spectrum of the SAR signal [1],

$E\left[\boldsymbol{s}(f) \cdot \boldsymbol{s}^{H}(f)\right]=\operatorname{diag}\left(\sigma_{i}^{2}(f)\right), \quad 1 \leq i \leq N$.

and, from (4),

$y_{k}(f)=\sum_{m=1}^{N} H_{k m}(f) \cdot s_{m}(f) \Rightarrow$

$E\left[\left|y_{k}(f)\right|^{2}\right]=\sum_{m=1}^{N} \sigma_{m}^{2}(f) \cdot\left|H_{k m}(f)\right|^{2}=\sum_{m=1}^{N} \sigma_{m}^{2}(f)$,

where $H_{i k}$ are the entries of $\boldsymbol{H}$ defined in (2) and the summation over $\sigma_{m}{ }^{2}$ is the signal power density $p_{s}(f)=E\left[\boldsymbol{s}^{H}(f) \cdot \boldsymbol{s}(f)\right]$ summed over all azimuth looks for a given frequency $f$ in $I_{1}$.

For the random variables $\beta_{k}$, taking the expectation using (15), the independence assumptions [15] and the fact that, for the amplitudes $\epsilon_{i} \sim N\left(0, \sigma_{\epsilon}^{2}\right) \Rightarrow E\left[\epsilon_{i}\right]=0, E\left[\epsilon_{i}^{2}\right]=\sigma_{\epsilon}^{2}$ yields $\sigma_{\beta}^{2}=E\left[\left|\beta_{k}\right|^{2}\right]=\sigma_{\epsilon}^{2}+2 \cdot E\left[1-\cos \left(\xi_{i}\right)\right]$,

It can be shown by applying the theorem for the probability density of a function of a random variable [15] and direct integration that, for $\xi \sim U\left(-\frac{\xi_{u}}{2}, \frac{\xi_{u}}{2}\right)$,

$E[\sin (\xi)]=0$;

$E[\cos (\xi)]=2 \cdot \frac{\sin \left(\frac{\xi_{u}}{2}\right)}{\xi_{u}}=\operatorname{sinc}\left(\frac{\xi_{u}}{2}\right)$.

Hence, (20) becomes

$\sigma_{\beta}^{2}=\left[\sigma_{\epsilon}^{2}+2 \cdot\left(1-\operatorname{sinc}\left(\frac{\xi_{u}}{2}\right)\right)\right]$.

Alternatively, using the small error model of (7), (20) can be simplified ${ }^{4}$ to

$\sigma_{\beta}^{2}=E\left[\left|\beta_{k}\right|^{2}\right]=\sigma_{\epsilon}^{2}+E\left[\left|\xi_{i}\right|^{2}\right]$,

regardless of the probability distribution, and for all $i$ (i.i.d).

\footnotetext{
${ }^{4}$ The small error model is useful if the phase errors are assumed to follow a distribution other than uniform, or show no particular structure. One example is a Gaussian error model, which is commonly adopted in engineering for error propagation from a large number of sources. If the errors are uniformly distributed, $E\left[\left|\xi_{i}\right|^{2}\right]=\xi_{u}^{2} / 12$ and (23) represents a Maclaurin expansion of (22), since $\sin (x) \cong x-\frac{x^{3}}{3 !} \Rightarrow 2 \cdot\left(1-\operatorname{sinc}\left(\frac{\xi_{u}}{2}\right)\right) \cong \frac{2}{6} \cdot\left(\frac{\xi_{u}}{2}\right)^{2}=\frac{\xi_{u}^{2}}{12}$.
}

Finally, (17) may be rewritten as

$$
\begin{aligned}
& \sigma_{\text {error }}^{2}(f)=p_{S}(f) \cdot \sigma_{\beta}^{2} \cdot \sum_{m=0}^{N} \sum_{k=0}^{N}\left|P_{m k}(f)\right|^{2} \\
& \sigma_{\text {error }}^{2}(f)=p_{S}(f) \cdot \sigma_{\beta}^{2} \cdot\|\boldsymbol{Q}(f)\|^{2}
\end{aligned}
$$

where $P_{i k}(f)$ denotes the entries of $\boldsymbol{P}(f)$ and thus $\|\boldsymbol{Q}(f)\|^{2}$ denotes the square of the Frobenius norm of the matrix $\boldsymbol{Q}(f)$, formed by taking element-wise the absolute value of $\boldsymbol{P}(f)_{N \times N}$. It is interesting to note that $\|\boldsymbol{Q}(f)\|^{2}$ is closely related to the SNR scaling factor of the reconstruction, defined in [6]:

$$
\begin{aligned}
\Phi_{\mathrm{REC}}\left(f_{\mathrm{PRF}}\right) & =\frac{N}{N \cdot f_{\mathrm{PRF}}} \cdot \sum_{k=1}^{N} \int_{-N \cdot f_{\mathrm{PRF}} / 2}^{N \cdot f_{\mathrm{PRF}} / 2}\left|P_{k}(f)\right|^{2} \cdot \mathrm{d} f \\
& =\frac{1}{f_{\mathrm{PRF}}} \cdot \sum_{k=1}^{N} \sum_{m=1}^{N} \int\left|P_{m k}(f)\right|^{2} \cdot \mathrm{d} f \\
& =\frac{1}{f_{\mathrm{PRF}}} \cdot \int\|\boldsymbol{Q}(f)\|^{2} \cdot \mathrm{d} f,
\end{aligned}
$$

meaning $\Phi_{\mathrm{REC}}\left(f_{\mathrm{PRF}}\right)$ is an average over frequency of $\|\boldsymbol{Q}(f)\|^{2}$. In the particular case $\boldsymbol{P}(f)=\boldsymbol{H}(f)^{-1}[6]$, this quantity admits a closed-form analytical expression exploiting the Van der Monde structure of $\boldsymbol{H}(f)$ for inversion [11], [14]. At this point, let an average over frequency be introduced as

$$
\begin{aligned}
& p_{\mathrm{amb}}=\frac{1}{f_{\mathrm{PRF}}} \cdot \int_{-N \frac{f_{\mathrm{PRF}}}{2}}^{-N \cdot \frac{f_{\mathrm{PRF}}}{2}+f_{\mathrm{PRF}}} E\left[\|\boldsymbol{\Delta}(f)\|^{2}\right] \cdot \mathrm{d} f \\
& p_{\mathrm{amb}}=\sigma_{\beta}^{2} \cdot \frac{1}{f_{\mathrm{PRF}}} \cdot \int_{-N \cdot \frac{f_{\mathrm{PRF}}}{2}}^{\frac{f_{\mathrm{P}}}{2}} \| f_{\mathrm{PRF}} \\
& p_{\mathrm{amb}}=\sigma_{\beta}^{2} \cdot\|\overline{\boldsymbol{Q}}\|^{2} \cdot p_{\text {signal }}^{\text {avg }}
\end{aligned}
$$

where $p_{\text {signal }}^{\text {avg }}$ is the average signal power density over all frequencies in $I_{1}$ and $\|\overline{\boldsymbol{Q}}\|^{2}$ is the average over frequency ${ }^{5}$ of $\|\boldsymbol{Q}\|^{2}$.

Using this result, one may define the error induced AASR ${ }^{6}$

$$
\begin{aligned}
& A A S R_{\text {errors }}=\frac{p_{\text {amb }}^{\text {avg }}}{p_{\text {signal }}^{\text {aig }}} \\
& A A S R_{\text {errors }}=\sigma_{\beta}^{2} \cdot\left(\sum_{i=1}^{N} \sum_{k=1}^{N}\left|p_{k i}\right|^{2}\right)
\end{aligned}
$$

$A A S R_{\text {errors }}=\sigma_{\beta}^{2} \cdot\|\overline{\boldsymbol{Q}}\|^{2}$,

where $\sigma_{\beta}^{2}$ is defined by either (22) or (23), depending on the phase error model. This is the final and main result of this section, and indicates that, interestingly, the SNR scaling factor also effectively scales the ambiguous energy due to residual channel imbalances. The $f_{\mathrm{PRF}}$ and the channel properties thus influence not only $A A S R_{\text {error-free }}$ but also the system's sensitivity, which is a new result following from the presented derivation.

\footnotetext{
${ }^{5}$ Due to the summation over the $N$ looks in $p_{s}(f)=E\left[\boldsymbol{s}^{H}(f) \cdot \boldsymbol{s}(f)\right]$, the integration interval of the result is $N \cdot f_{\mathrm{PRF}}$, which applies to the range compressed image. The integration over frequency to determine $\|\overline{\boldsymbol{Q}}\|^{2}$ and the signal power should be restricted to the processed bandwidth to account for the azimuth matched filter.

${ }^{6}$ The AASR may be interpreted in a general sense as the well-known integrated sidelobe ratio (ISLR), used as a quality parameter for radar images. Recall from (14) that the ambiguous energy arises both from the excess Doppler bandwidth with respect to $N \cdot f_{\mathrm{PRF}}$ (azimuth antenna pattern) and from the channel model mismatch, which leads to reconstruction errors and reduced ambiguity suppression in comparison to the error-free case.
} 


\section{Simulation Results}

In this section, the analytical AASR model is verified by means of a Monte Carlo simulation of a C-band multichannel SAR system. The reconstruction (in this example, $\boldsymbol{P}(f)=\boldsymbol{H}(f)^{-1}[6]$ ) of a point target at a look angle $\theta_{\text {look }}=32.5^{\circ}$ is performed for 1000 realizations of the errors drawn from the appropriate distributions (i.e. $U\left[-\frac{\xi_{u}}{2}, \frac{\xi_{u}}{2}\right]$ for phase errors and $N\left(0, \sigma_{\epsilon}^{2}\right)$ for amplitude errors) and the average AASR (estimated for each realization using the impulse response based method of [16]) is taken as an estimation of the expected values. This is repeated for different values of the corresponding error distribution parameters $\left(\xi_{u}\right.$ or $\left.\sigma_{\epsilon}^{2}\right)$ to measure the performance degradation as a function of the error level. The phase and amplitude errors are analyzed independently to allow a better visualization of the results, and the simulated signals are noiseless. Given the relevance of the noise scaling factor $\|\overline{\boldsymbol{Q}}\|^{2}$ for the sensitivity with respect to errors in (27), two different scenarios with two different values of $f_{\mathrm{PRF}}$ are considered, to illustrate the behavior both for favorable and unfavorable sampling conditions. The system parameters are summarized in TABLE I. On transmit, phase-spoiling is used to widen the patterns.

TABLE I

SiMULATION SCENARIO PARAMETERS

\begin{tabular}{lll}
\hline \hline \multicolumn{3}{c}{ Relevant System Parameters (Azimuth) } \\
\hline \multicolumn{1}{c}{ Symbol } & \multicolumn{1}{c}{ Quantity } & Value \\
\hline$h_{\text {orbit }}$ & Orbit height & $700 \mathrm{~km}$ \\
$\lambda$ & Wavelength & $5.54 \mathrm{~cm}$ \\
$l_{\mathrm{az}}$ & Antenna length in azimuth & $12.8 \mathrm{~m}$ \\
$N_{\mathrm{az}}$ & Number of azimuth channels & 8 \\
$\Delta x_{\mathrm{az}}$ & (Physical) Spacing of azimuth channels & $1.6 \mathrm{~m}$ \\
$B w_{\mathrm{proc}}$ & Processed bandwidth & $5773 \mathrm{~Hz}$ \\
$\delta_{a z}$ & Goal azimuth resolution & $1 \mathrm{~m}$
\end{tabular}

The first scenario regards the uniform sampling case with $f_{\mathrm{PRF}}=1172 \mathrm{~Hz}$, and presents a multichannel PRF of $N \cdot f_{\mathrm{PRF}}=9376 \mathrm{~Hz}$. This means considerable oversampling with respect to the signal bandwidth. As expected, error-free reconstruction leads to a low $A A S R_{\text {error-free }}=-31 \mathrm{~dB}$, at an azimuth resolution of $1.0 \mathrm{~m}$. The corresponding impulse response is depicted in Fig. 2 (a). Notice that the abscissas are the instant Doppler frequencies rather the azimuth spatial coordinates, to highlight the origin of the ambiguous components. The ambiguities at integer multiples of $f_{\mathrm{PRF}}$, highlighted in blue, show very low levels, with the exception of the ones at $\pm 9376 \mathrm{~Hz}$, which correspond to the azimuth ambiguities of the error-free case. In contrast, they are visibly higher in Fig. 2 (b), which is the result of reconstruction with channels affected by (a realization of) phase errors drawn from a uniform distribution in the interval $\left[-5^{\circ}, 5^{\circ}\right]$ (i.e., $\xi_{u}=10^{\circ}$ ).

Fig. 3 (a) and (b) illustrate the histograms of the AASR over the Monte Carlo trials, respectively for varying $\xi_{u}$ (phase errors) and $\sigma_{\epsilon}$. The expected value of the AASR for each value of the respective distribution parameter is highlighted by vertical dashed lines in the same color of the histograms. It is clear that the AASR rises quickly from the error-free value for small errors, but the histograms migrate more slowly towards larger AASR levels for higher error magnitudes (cf. distance between vertical lines in Fig. 3 (a)).

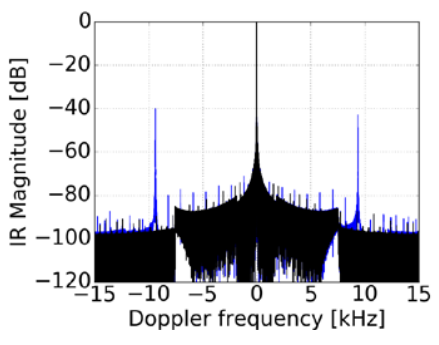

(a)

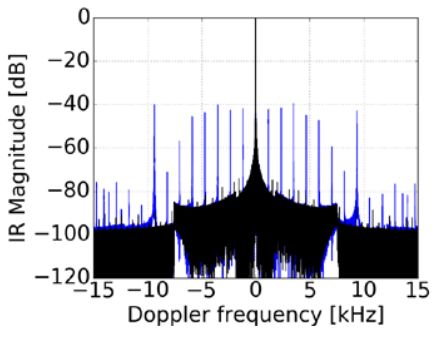

(b)
Fig. 2: Example of focused impulse responses after reconstruction with and without channel imbalances, for $f_{\mathrm{PRF}}=1172 \mathrm{~Hz}$. (a) Error-free reconstruction, with very low AASR. (b) Reconstruction with a realization of uniform phase errors drawn from $\left[-5^{\circ}, 5^{\circ}\right]$. Ambiguites are visible in blue.

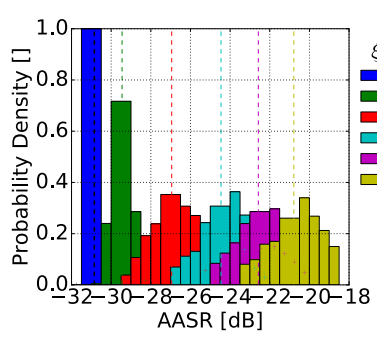

(a)

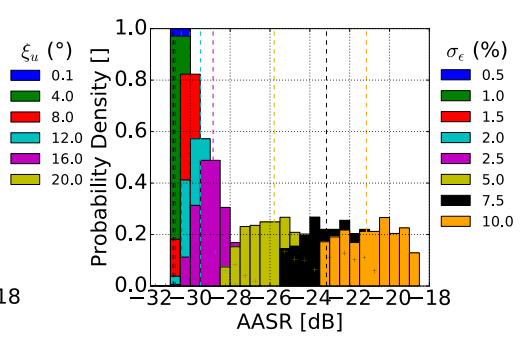

(b)
Fig. 3: Monte Carlo simulation of reconstruction, for $f_{\mathrm{PRF}}=1172 \mathrm{~Hz}$. (a) AASR histograms over Monte Carlo trials, parametrized by $\xi_{u}$. (b) AASR histograms parametrized by $\sigma_{\epsilon}$. The black vertical dashed lines indicate $A A S R_{\text {error-free }}$ and the color-coded ones, the mean of each histogram.

The second scenario was designed to achieve error-free AASR levels of $-25 \mathrm{~dB}$. The degraded performance is obtained by reducing the PRF to $f_{\mathrm{PRF}}=1149 \mathrm{~Hz}$, which does not change the achieved resolution.

The AASR induced by phase and amplitude errors, as estimated by the Monte Carlo approach, is shown as a function of the distribution parameters $\xi_{u}$ and $\sigma_{\epsilon}$ in Fig. 4, for both $f_{\mathrm{PRF}}$ scenarios. The error bars show an interval of one standard deviation centered on the means of the Monte Carlo simulations, which correspond to the position of the vertical dashed lines in Fig. 3.

The analytical model prediction calculated from (14), (27) is also plotted, showing very good agreement to the Monte Carlo simulations, especially for smaller errors. The maximum deviation between the curves is overall smaller than $0.15 \mathrm{~dB}$. The expected AASR levels (red curve in Fig. 4) are dominated by the nominal levels $A A S R_{\text {error-free }}$ for small values of $\xi_{u}$, and thus the initial sensitivity of the AASR to errors is low. For the first $f_{\mathrm{PRF}}$ scenario - in Fig. 4 (a), (b) - the sensitivity increases for intermediate errors, however saturation effects result in a slightly reduced sensitivity for larger imbalances. The level of $-25 \mathrm{~dB}$, considered a threshold of the maximum acceptable residual ambiguity ratio, is reached for $\xi_{u} \cong 10.9^{\circ}$ or $\sigma_{\epsilon} \cong 5.5 \%$. In the second scenario - Fig. 4 (c), (d) owing to the higher $A A S R_{\text {error-free }}$, the impact of errors is only visible for larger imbalances and the sensitivity does not change appreciably for the interval of error parameters considered in the analysis.

Finally, to illustrate the usefulness of the presented error model in deriving requirements, a mapping of the total AASR (with errors) as a function of the initial error-free AASR and the phase error distribution range $\xi_{u}$ is shown in Fig. 5. In this example, 
$\|\overline{\mathbf{Q}}\|^{2}=1$, meaning uniform sampling is considered, and no amplitude errors occur $\left(\sigma_{\epsilon}^{2}=0\right)$, which can be understood as a bestcase analysis of the behavior of the phase-error induced AASR. The contour lines of the total AASR illustrate how a trade-off between the maximum residual phase error (which translates into hardware and calibration requirements) and the sampling conditions (a function of element spacing and PRF) may be used to achieve a specified performance level in the system design. The results illustrate on the one hand that the error contribution quickly dominates for operating points in which the error-free level is very low, making them hardly achievable in practice. On the other hand, some margin for the error contribution is seen to be required, according to the hardware's and calibration system capabilities in terms of the achievable residual error magnitude.

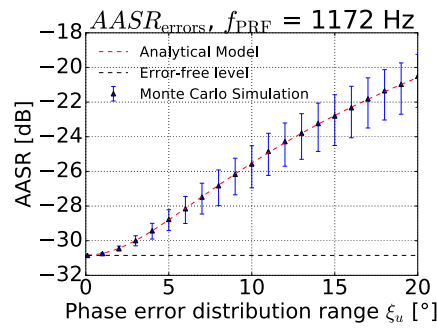

(a)

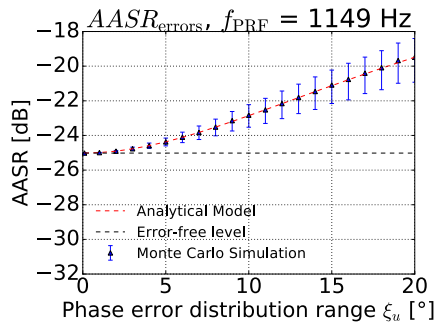

(c)

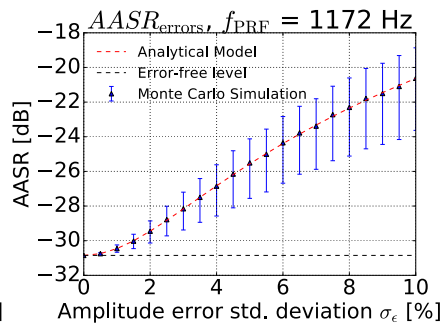

(b)

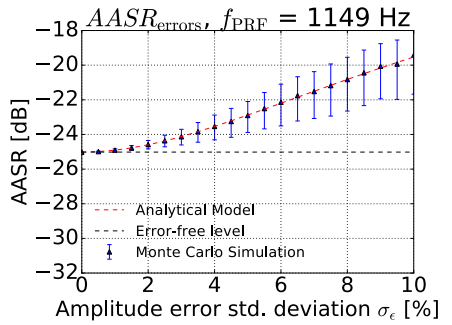

(d)
Fig. 4: AASR as function of phase error uniform distribution range $\xi_{u}(\mathrm{a}, \mathrm{c})$ and amplitude error standard deviation $\sigma_{\epsilon}(\mathrm{b}, \mathrm{d})$, for $f_{\mathrm{PRF}}=1172 \mathrm{~Hz}(\mathrm{a}, \mathrm{b})$ and $f_{\mathrm{PRF}}=1149 \mathrm{~Hz}(\mathrm{c}, \mathrm{d})$. The blue dots show the average derived from the Monte Carlo simulations, whereas the error bar shows an interval of one standard deviation centered on the mean. The red dashed line shows the result of the analytical prediction, with the error-free level (respectively $-31 \mathrm{~dB}$ and $-25 \mathrm{~dB}$ for the two scenarios) indicated by a horizontal black dashed line.

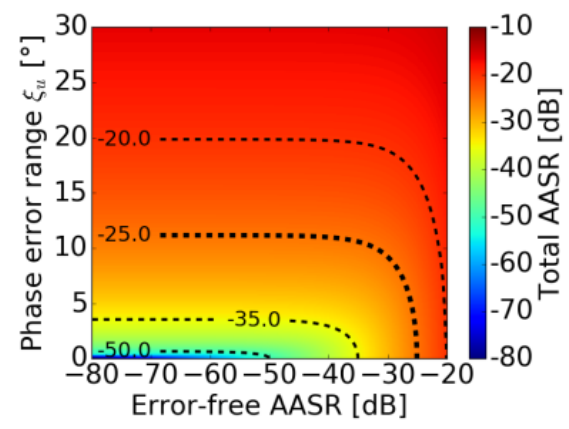

Fig. 5: 2D mapping of total AASR as a function of the error-free AASR (abscissa) and the phase error distribution parameter $\xi_{u}$ (ordinate), with contour lines indicating total AASR level boundaries in $\mathrm{dB}$.

\section{FINAL REMARKS AND DISCUSSION}

The paper presented a residual phase-amplitude error model for a system with multiple receive channels in azimuth and derived mathematically an analytical model for the error's impact on the ambiguous energy found in the reconstructed image, measured by means of the AASR performance parameter. An interesting new result is that the well-known SNR scaling parameter also plays an important role in the scaling of the error-induced ambiguous energy. The sampling conditions are therefore also relevant for the robustness of the processing approach with respect to errors in the channel's transfer functions.

The model was validated to a good extent by means of Monte Carlo simulations of the errors, following the appropriate probability distribution assumed in the derivation. This leads to the conclusion that the assumed approximations are reasonable and no appreciable deviations are expected from the computationally more costly Monte Carlo approach. The model thus presents itself as a simple and effective alternative to estimate the impacts of residual errors and may be used as a design aid for e.g. establishing radar electronics and calibration requirements for the next generation of multichannel spaceborne SAR systems.

\section{REFERENCES}

[1] J. C. Curlander and R. N. McDonough. "Synthetic Aperture Radar: Systems and Signal Processing", New York: Wiley, 1991.

[2] G. Krieger et al., "SIMO and MIMO System Architectures and Modes for High-Resolution Ultra-Wide-Swath SAR Imaging," in 11th European Conference on Synthetic Aperture Radar (EUSAR), pp. 1-6., Hamburg, 2016.

[3] M. Younis et al., "Techniques and Modes for Multi-Channel SAR Instruments," in 11th European Conference on Synthetic Aperture Radar (EUSAR), pp. 1-6, Hamburg, 2016.

[4] A. Freeman et al., "The "Myth" of the minimum SAR antenna area constraint," IEEE Trans. Geosci. Remote Sens., vol. 38, no. 1, pp. 320-324, 2000.

[5] G. Krieger, N. Gebert and A. Moreira, "Unambiguous SAR signal reconstruction from nonuniform displaced phase center sampling," in IEEE Geosci. Remote Sens. Lett., vol. 1, no. 4, pp. 260-264, 2004.

[6] N. Gebert, G. Krieger, and A. Moreira. "Digital beamforming on receive: Techniques and optimization strategies for high resolution wide-swath SAR imaging", in IEEE Trans. Aerosp. Electron. Syst., vol. 45, pp. 564-592, 2009.

[7] G. Adamiuk, C. Schaefer, C. Fischer and C. Heer. "SAR Architectures based on DBF for C- and X-band applications", 10th European Conference on Synthetic Aperture Radar (EUSAR), pp. 1-4, 2014.

[8] M. Ludwig, J-C. Angevain, C. Buck and D. Petrolati. "Multi-channel Synthetic Aperture Radar in Europe," in 2017 IEEE International Geoscience and Remote Sensing Symposium, Fort Worth, Texas, 2017.

[9] A. Papoulis, "Generalized Sampling Expansion", IEEE Trans. on Circuits and Systems, vol. 24, no. 11, pp. 652-654, 1977.

[10] P. Laskowski, F. Bordoni and M. Younis, "Multi-channel SAR performance analysis in the presence of antenna excitation errors," 2013 14th International Radar Symposium (IRS), Dresden, pp. 491-496, 2013.

[11] D. Seidner and M. Feder. "Vector Sampling Expansion", IEEE Trans. Signal Process., vol. 48, no. 5, pp. 1401-1416, 2000.

[12] I. Sikaneta, C.H. Gierull, and D. Cerutti-Maori. "Optimum signal processing for multichannel SAR: with application to high-resolution wide-swath imaging", IEEE Trans. Geosci. Remote Sens., vol. 52, no. 10, pp. 6095-6109, 2014.

[13] D. Cerutti-Maori, I. Sikaneta, J. Klare and C. H. Gierull, "MIMO SAR Processing for Multichannel High-Resolution Wide-Swath Radars," in IEEE Trans. Geosci. Remote Sens., vol. 52, no. 8, pp. 5034-5055, 2014.

[14] D. Seidner and M. Feder. "Noise amplification of periodic nonuniform sampling”, IEEE Trans. Signal Process., vol. 48, no. 1, pp. 275-277, 2000.

[15] A. Papoulis and S. U. Pillai. "Probability, random variables, and stochastic processes", Chapter 5, New York: McGraw Hill, 2002.

[16] M. Villano, G. Krieger and A. Moreira "Ambiguities and image quality in staggered SAR" in 5th Asia-Pacific Conference on Synthetic Aperture Radar (APSAR), Singapore, pp. 204-209, 2015. 